\title{
Convolution Kernel
}

National Cancer Institute

\section{Source}

National Cancer Institute. Convolution Kernel. NCI Thesaurus. Code C41832.

An algorithm used to reconstruct imaging data. Convolution kernels can be used to filter images to smooth them and reduce noise or to enhance edges or other image features. 